\title{
Risk Factors for Postoperative Recurrence in Korean Patients with Crohn's Disease
}

\begin{abstract}
Sung Bae Kim ${ }^{1}$, Jae Hee Cheon ${ }^{1}$, Jae Jun Park ${ }^{1}$, Eun Soo Kim ${ }^{2}$, Seong Woo Jeon ${ }^{2}$, Sung-Ae Jung ${ }^{3}$, Dong II Park ${ }^{4}$, Chang Kyun Lee ${ }^{5}$, Jong Pil Im ${ }^{6}$, You Sun Kim ${ }^{7}$, Hyun Soo Kim ${ }^{8}$, Jun Lee ${ }^{9}$, Chang Soo Eun ${ }^{10}$, Jeong Mi Lee ${ }^{11}$, Byung Ik Jang ${ }^{12}$, and Geom Seog Seo ${ }^{13}$

${ }^{1}$ Department of Internal Medicine, Institute of Gastroenterology, Yonsei University College of Medicine, Seoul, ${ }^{2}$ Department of Internal Medicine, School of Medicine, Kyungpook National University, Daegu, ${ }^{3}$ Department of Internal Medicine, Ewha Womans University School of Medicine, ${ }^{4}$ Department of Internal Medicine, Kangbuk Samsung Hospital, Sungkyunkwan University School of Medicine, ${ }^{5}$ Center for Crohn's and Colitis, Department of Gastroenterology, Kyung Hee University College of Medicine, ${ }^{6}$ Department of Internal Medicine and Liver Research Institute, Seoul National University College of Medicine, ${ }^{7}$ Department of Internal Medicine, Seoul Paik Hospital, Inje University College of Medicine, Seoul, ${ }^{8}$ Division of Gastroenterology, Department of Internal Medicine, Chonnam National University Medical School, ${ }^{9}$ Department of Internal Medicine, Chosun University College of Medicine, Gwangju, ${ }^{10}$ Department of Internal Medicine, Hanyang University Guri Hospital, Guri, ${ }^{11}$ Department of Public Health, Wonkwang University Graduate School, Iksan, ${ }^{12}$ Department of Internal Medicine, Yeungnam University College of Medicine, Daegu, and ${ }^{13}$ Department of Internal Medicine, Digestive Disease Research Institute, Wonkwang University College of Medicine, Iksan, Korea
\end{abstract}

Background/Aims: A considerable number of patients with Crohn's disease still need intestinal resection surgery. Postoperative recurrence is an important issue in Crohn's disease management, including the selection of high-risk patients. Eastern Asian patients showed several differences from Caucasian patients. Therefore, we investigated the postoperative surgical recurrence outcome and identified risk factors in Korean patients. Methods: Clinical data of 372 patients with Crohn's disease who underwent first intestinal resection between January 2004 and August 2014 at 14 hospitals in Korea were retrospectively reviewed. Results: Over the follow-up period, 50 patients (17.1\%) showed surgical recurrence. The cumulative surgical recurrence rate was $6.5 \%$ at 1 year and $15.4 \%$ at 7 years. Age under $16(p=0.011$; hazard ratio [HR], 5.136; 95\% confidence interval [Cl], 1.576 to 16.731 ), colonic involvement ( $p=0.023$; HR , 2.011; 95\% $\mathrm{Cl}, 1.102$ to 3.670), and the presence of perianal disease at surgery ( $p=0.008$; HR, 2.239; 95\% Cl, 1.236 to 4.059) were independent risk factors associated with surgical recurrence. Postoperative thiopurine treatment $(p=0.002$; HR, 0.393; $95 \% \mathrm{Cl}, 0.218$ to 0.710 ) was a protective factor for surgical recurrence. Conclusions: Among the disease characteristics at surgery, younger age, colonic location, and perianal le- sions were independent risk factors for surgical recurrence. Postoperative thiopurine treatment significantly reduced the incidence of surgical recurrence. (Gut Liver 2020;14:331337)

Key Words: Crohn disease; Recurrence; Surgery; Risk factors; Thiopurine

\section{INTRODUCTION}

Crohn's disease (CD) is a chronic, progressive, and disabling inflammatory bowel disease with uncertain etiology and pathogenesis. CD can affect any part of the gastrointestinal tract with systemic manifestations. ${ }^{1}$ The natural course of the $\mathrm{CD}$ is characterized by relapse and remissions. Advancement in medical treatment, such as immunomodulators and anti-tumor necrosis factor (anti-TNF)- $\alpha$ antibody, has changed the treatment strategy for $\mathrm{CD}^{2}{ }^{2}$ Using anti-TNF- $\alpha$ antibody and immunomodulator has achieved mucosal healing that led to long-term maintenance of remission and lowering surgery rate. In a Danish cohort study, 5-year cumulative probability of first major surgery decreased from $44.7 \%$ in a cohort (1979 to 1986 ) to $19.6 \%$ in a cohort (2003 to 2011) for CD. ${ }^{3}$

Correspondence to: Geom Seog Seo ${ }^{\mathrm{a}}$ and Byung Ik Jang ${ }^{\mathrm{b}}$

${ }^{a}$ Department of Internal Medicine, Digestive Disease Research Institute, Wonkwang University College of Medicine, 460 Iksan-daero, Iksan 54538, Korea

Tel: +82-63-859-2565, Fax: +82-63-855-2025, E-mail: medsgs@wonkwang.ac.kr

${ }^{b}$ Division of Gastroenterology and Hepatology, Department of Internal Medicine, Yeungnam University College of Medicine, 170 Hyeonchung-ro, Nam-gu, Daegu 42415, Korea

Tel: +82-53-620-3831, Fax: +82-53-654-8386, E-mail: jbi@med.yu.ac.kr

Received on February 27, 2019. Revised on May 16, 2019. Accepted on May 31, 2019. Published online September 26, 2019. pISSN 1976-2283 eISSN 2005-1212 https://doi.org/10.5009/gnl19085

(a) This is an Open Access article distributed under the terms of the Creative Commons Attribution Non-Commercial License (http://creativecommons.org/licenses/by-nc/4.0) which permits unrestricted non-commercial use, distribution, and reproduction in any medium, provided the original work is properly cited. 
Nevertheless, considerable patients with CD need surgical intervention including a requirement for intestinal resection even in the biologic agent treatment era. ${ }^{4}$ Intestinal resection for CD is limited to maintain the complete remission status due to high recurrence rate. More than half of the patients experience a clinical recurrence within 5 years and surgical recurrence within 10 years. ${ }^{5}$ Therefore, the prevention of postoperative recurrence, especially the need for repeated intestinal resection after the first surgery is a crucial issue.

Selecting high-risk patients for CD recurrence is an important problem. Smoking, perforating disease phenotype, and previous intestinal resection have been suggested from previous studies as factors that predict earlier postoperative recurrence. ${ }^{6,7}$ A prior randomized clinical trial showed that treatment according to clinical risk factors of recurrence, early colonoscopy and treatment step-up for recurrence is better for prevention of $\mathrm{CD}$ recurrence. $^{8}$

However, most of the previous studies regarding postoperative recurrence in $\mathrm{CD}$ were performed in Western nations and mainly Caucasians because of relatively lower incidence CD in Eastern Asia. Clinical characteristics such as age at diagnosis, cigarette smoking, disease location, behavior, and treatment strategy showed differences between the East and the West in studies. ${ }^{9,10}$ Indeed, factors that predict postoperative recurrence are still unclear and the data are lacking, especially in Eastern Asian countries. Previous studies were limited in terms of small patient number, single hospital patients or lack of clinical and medication data. ${ }^{11-13}$ Consequently, research of postoperative recurrence is needed to target Eastern Asian patients with CD.

Therefore, we investigated postoperative surgical recurrence outcomes and identified the risk factors associated with postoperative recurrence after intestinal resection surgery in Korean patients with $\mathrm{CD}$ through a multicenter setting study.

\section{MATERIALS AND METHODS}

\section{Patients}

Clinical data of 372 patients with CD who underwent first intestinal resection due to CD from January 2004 to August 2014 at 14 hospitals in Korea were retrospectively reviewed. Surgical intervention without intestinal resection such as operation limited in appendectomy, perianal procedure, and strictureplasty was not included as intestinal resection surgery. Surgical recurrence was defined as additional intestinal resection owing to CD, except surgery-related complications.

Patients with the following were excluded: follow-up period less than 1 year after intestinal resection $(n=43)$, and patients with insufficient clinical, surgical information $(n=37)$. After excluding 80 patients based on our exclusion criteria, a total of 292 patients were finally selected.

This study was approved by the Institutional Review Board of Wonkwang University Hospital in Iksan, Korea (IRB number:

\section{WKUH 201401-HRE-007).}

\section{Primary and secondary outcomes}

The primary outcome was to investigate the risk factors of postoperative surgical recurrences in Korean patients with CD. The secondary outcome was to evaluate surgical recurrence rates according to risk factors.

\section{Statistical analysis}

Data are expressed as the median (interquartile range), number, or number (\%), as appropriate. Factors associated with surgical recurrence were investigated using the Kaplan-Meier analysis and Cox regression. Any variables identified as significant $(\mathrm{p}<0.05)$ in the univariate analysis were included in the multivariate analysis.

For comparison of the cumulative probability of surgical recurrences, Kaplan-Meier estimator survival analysis was used. For comparing outcomes, the chi-square test or Fisher exact test was used for categorical data, and the Student t-test was used for continuous variables. Data analysis was performed using SPSS software version 18.0 (SPSS Inc., Chicago, IL, USA). A two-sided p-value $<0.05$ was considered statistically significant.

\section{RESULTS}

\section{Baseline characteristics}

Baseline characteristics of the patients are summarized in Table 1. The median age at diagnosis was 25.6 years and at surgery was 29.6 years, respectively. Based on Montreal classification, the majority of the patients presented with ileocolonic location (L3, 46.2\%) and non-stricturing, non-penetrating behavior (B1, 53.1\%) at diagnosis. At the time of surgery, the patients' disease phenotype changed, resulting in the most common location to terminal ileum (L1, 53.1\%) and behavior to penetrating type (B3. 66.4\%). Most of the patients were diagnosed between ages of 17 and 40 ( $n=196,67.1 \%)$. Patients with age under 16 years at diagnosis and at surgery were $63(21.6 \%)$ and 34 (11.6\%). At diagnosis, 51 patients (17.5\%) were current smokers and 11 patients (21.6\%) stopped smoking before their first intestinal resection surgery. Perianal diseases were presented in 135 patients (46.2\%) before their first intestinal resection surgery.

\section{Medical management}

Patients' medical therapy histories before and after surgery is described in Table 2. The median duration from diagnosis to surgery was 29.0 months and median follow-up periods after intestinal resection was 46.0 months. Thiopurine (azathioprine or 6-mercaptopurine) was used in 161 patients (55.1\%) before surgery and 207 patients (70.9\%) after first intestinal resection surgery. The number of patients using anti-TNF agents (infliximab or adalimumab) before and after intestinal resection sur- 
gery was 58 (19.9\%) and 93 (31.8\%). In preoperative anti-TNF users, 35 patients (60.3\%) reused anti-TNF agents after bowel resection.

\section{Postoperative surgical recurrence}

Postoperative surgical recurrence outcome is showed in Table 3. Fifty patients (17.1\%) received second intestinal resection surgery due to $\mathrm{CD}$. The cumulative surgical recurrence rate increased from $6.5 \%$ at 1 year to $15.4 \%$ at 7 years.

Table 1. Baseline Characteristics at Diagnosis and Surgery

\begin{tabular}{|c|c|c|}
\hline Variable & $\begin{array}{c}\text { Diagnosis } \\
(\mathrm{n}=292)\end{array}$ & $\begin{array}{l}\text { Surgery } \\
(\mathrm{n}=292)\end{array}$ \\
\hline \multicolumn{3}{|l|}{ Sex } \\
\hline Male & $198(67.8)$ & $198(67.8)$ \\
\hline Female & $94(32.2)$ & $94(32.2)$ \\
\hline Age, yr & $25.6(17.0-32.0)$ & $29.6(21.0-35.8)$ \\
\hline A1 (16) & 63 (21.6) & 34 (11.6) \\
\hline A2 (17-40) & $196(67.1)$ & 207 (70.9) \\
\hline A3 $(>40)$ & $33(11.3)$ & $51(17.5)$ \\
\hline \multicolumn{3}{|l|}{ Location } \\
\hline L1 (ileal) & $116(39.7)$ & $155(53.1)$ \\
\hline L2 (colonic) & $37(12.7)$ & $41(14.0)$ \\
\hline L3 (ileocolonic) & $135(46.2)$ & $92(31.5)$ \\
\hline L4 (isolated upper disease) & $4(1.4)$ & $4(1.4)$ \\
\hline \multicolumn{3}{|l|}{ Behavior } \\
\hline $\begin{array}{l}\text { B1 (non-stricturing, } \\
\text { non-penetrating) }\end{array}$ & $130(44.5)$ & $42(14.4)$ \\
\hline B2 (stricturing)* & $102(34.9)$ & $86(29.5)$ \\
\hline B3 (penetrating) $^{\dagger}$ & $70(24.0)$ & $194(66.4)$ \\
\hline $\mathrm{P}$ (perianal disease) & $115(39.4)$ & $135(46.2)$ \\
\hline \multicolumn{3}{|l|}{ Current smoking } \\
\hline Current smoker & $51(17.5)$ & 40 (13.7) \\
\hline Ex-smoker + non-smoker & $241(82.5)$ & $252(86.3)$ \\
\hline CD diagnosed after surgery & $49(16.8)$ & - \\
\hline Family history of IBD & $8(2.7)$ & - \\
\hline
\end{tabular}

Data are presented as number (\%) or median (range).

$\mathrm{CD}$, Crohn's disease; IBD, inflammatory bowel disease.

*Stricturing behavior, including the B2 (stricturing) and B2+B3 (stricturing and penetrating) phenotypes; ${ }^{\dagger}$ Penetrating behavior, including the $\mathrm{B} 3$ (penetrating) and $\mathrm{B} 2+\mathrm{B} 3$ (stricturing and penetrating) phenotypes.

\section{Factors associated with surgical recurrence}

To determine independent factors associated with surgical recurrence, variables including baseline characteristics and type of medication were analyzed. Among the variables, age at surgery $(\mathrm{p}=0.003)$, colonic involvement $(\mathrm{p}=0.009)$, penetrating phenotype $(\mathrm{p}=0.042)$, perianal disease $(\mathrm{p}=0.014)$, and postoperative thiopurine use $(p=0.014)$ were associated with surgical recurrence in univariate analysis. In multivariate analysis, age under 16 at surgery $(\mathrm{p}=0.011$; hazard ratio [HR], 5.136; 95\% confidence intervals [CI], 1.576 to 16.731), colonic involvement at surgery $(\mathrm{p}=0.023 ; \mathrm{HR}, 2.011 ; 95 \%$ CI, 1.102 to 3.670), and presence of perianal disease before surgery ( $p=0.008$; HR, 2.239; 95\% CI, 1.236 to 4.059 ) were independent risk factors associated with surgical recurrence. Using postoperative thiopurine medication ( $\mathrm{p}=0.002$; HR, 0.393; 95\% CI, 0.218 to 0.710 ) was a protective factor from surgical recurrence (Table 4).

\section{Surgical recurrence outcome according to risk factors}

High-risk group patients and low-risk group patients were stratified according to the number of risk factors that showed significant in multivariate analysis. Patients who had one risk factor were not different from those without risk factors. However, patients who had two or three risk factors showed higher rate of surgical recurrence (Table 5). There was no difference in follow-up duration between the two groups. Time to surgical recurrence was 84.3 months in patients with the high-risk group and 103.4 months in the low-risk group (Table 6).

Table 2. Crohn's Disease Medical Therapy ( $\mathrm{n}=292)$

\begin{tabular}{lcc}
\hline \multicolumn{1}{c}{ Variable } & Before surgery & After surgery \\
\hline Follow-up duration, mo & $29.0(1.0-74.8)$ & $46.0(24.0-72.0)$ \\
Surgical recurrence & - & $50(17.1)$ \\
Medication & & \\
5-ASA & $225(77.1)$ & $260(89.0)$ \\
Corticosteroid & $169(57.9)$ & $99(33.9)$ \\
Thiopurine & $161(55.1)$ & $207(70.9)$ \\
Anti-TNF & $58(19.9)$ & $93(31.8)$ \\
$\quad$ Infliximab & $52(17.8)$ & $78(26.7)$ \\
$\quad$ Adalimumab & $10(3.4)$ & $21(7.2)$ \\
$\quad$ Infliximab and adalimumab & $4(1.4)$ & $8(2.7)$
\end{tabular}

Data are presented as median (range) or number (\%).

ASA, aminosalicylic acid; TNF, tumor necrosis factor.

Table 3. Surgical Recurrence Outcome

\begin{tabular}{lcccccrr}
\hline \multirow{2}{*}{ Variable } & \multicolumn{7}{c}{ Time after first bowel resection surgery (yr) } \\
\cline { 2 - 8 } & 1 & 2 & 3 & 4 & 5 & 6 & 7 \\
\hline Surgical recurrence & $19 / 292(6.5)$ & $23 / 221(10.4)$ & $30 / 175(17.1)$ & $30 / 135(22.2)$ & $21 / 105(20.0)$ & $16 / 74(21.6)$ & $14 / 55(25.5)$ \\
Cumulative surgical recurrence & $19 / 292(6.5)$ & $25 / 292(8.5)$ & $34 / 292(11.6)$ & $40 / 292(13.7)$ & $41 / 292(14.0)$ & $43 / 292(14.7)$ & $45 / 292(15.4)$ \\
\hline
\end{tabular}

Data are presented as number/number (\%). 
334 Gut and Liver, Vol. 14, No.3, May 2020

Table 4. Independent Factors Associated with Postoperative Surgical Recurrence

\begin{tabular}{|c|c|c|c|c|c|c|}
\hline \multirow{2}{*}{ Variables } & \multicolumn{3}{|c|}{ Univariate } & \multicolumn{3}{|c|}{ Multivariate } \\
\hline & HR & $95 \% \mathrm{CI}$ & p-value & $\mathrm{HR}$ & $95 \% \mathrm{CI}$ & p-value \\
\hline Age at Surgery & & & 0.003 & & & 0.011 \\
\hline$\leq 16$ & 5.933 & $1.8492-19.108$ & & 5.136 & $1.576-16.731$ & \\
\hline $17-40$ & 2.483 & $0.876-7.058$ & & 2.169 & $0.759-6.204$ & \\
\hline$\geq 41$ & Reference & & & Reference & & \\
\hline Sex & 1.317 & $0.744-2.334$ & 0.342 & & & \\
\hline Family history & 0.653 & $0.090-4.740$ & 0.850 & & & \\
\hline Previous appendectomy & 1.068 & $0.500-2.280$ & 0.864 & & & \\
\hline Diagnosis by initial surgery & 1.716 & $0.723-4.074$ & 0.215 & & & \\
\hline Disease location at surgery & & & 0.009 & & & 0.023 \\
\hline Ileum & Reference & & & Reference & & \\
\hline Colon* & 2.173 & $1.196-3.951$ & & 2.011 & $1.102-3.670$ & \\
\hline \multicolumn{7}{|l|}{ Disease behavior at surgery } \\
\hline Non-stricturing, non-penetrating & 0.381 & $0.118-1.225$ & 0.092 & & & \\
\hline Stricturing $^{\dagger}$ & 0.793 & $0.419-1.501$ & 0.475 & & & \\
\hline Penetrating $^{\ddagger}$ & 2.019 & $1.009-4.041$ & 0.042 & & & 0.131 \\
\hline Perianal disease at surgery & 2.017 & $1.137-3.576$ & 0.014 & 2.239 & $1.236-4.059$ & 0.008 \\
\hline Current smoking at surgery & 1.290 & $0.509-3.268$ & 0.589 & & & \\
\hline Emergent surgery & 0.954 & $0.501-1.817$ & 0.885 & & & \\
\hline \multicolumn{7}{|l|}{ Preoperative Medication } \\
\hline 5-ASA & 1.015 & $0.528-1.953$ & 0.964 & & & \\
\hline Corticosteroid & 0.785 & $0.450-1.371$ & 0.393 & & & \\
\hline Thiopurine & 1.191 & $0.674-2.105$ & 0.546 & & & \\
\hline Anti-TNF & 0.852 & $0.399-1.820$ & 0.679 & & & \\
\hline \multicolumn{7}{|l|}{ Postoperative Medication } \\
\hline 5-ASA & 0.497 & $0.221-1.116$ & 0.083 & & & \\
\hline Corticosteroid & 1.092 & $0.613-1.943$ & 0.765 & & & \\
\hline Thiopurine & 0.496 & $0.280-0.878$ & 0.014 & 0.393 & $0.218-0.710$ & 0.002 \\
\hline Anti-TNF & 0.698 & $0.378-1.290$ & 0.025 & & & \\
\hline
\end{tabular}

HR, hazard ratio; CI, confidence interval; ASA, aminosalicylic acid; TNF, tumor necrosis factor.

${ }^{*}$ Colonic involvement, including L2 (colonic) and L3 (ileocolonic); ${ }^{\dagger}$ Stricturing behavior, including the B2 (stricturing) and B2+B3 (stricturing and penetrating) phenotypes; ' Penetrating behavior, including the B3 (penetrating) and B2+B3 (stricturing and penetrating) phenotypes.

Table 5. Surgical Recurrence Outcome According to Nonmodifiable Risk Factors

\begin{tabular}{ccccc}
\hline No. of risk factors* & No. (\%) & HR & 95\% CI & p-value \\
\hline 0 & $90(30.8)$ & & & $0.945-6.803$ \\
1 & $113(38.7)$ & 2.536 & $1.532-11.027$ & 0.065 \\
2 & $78(26.7)$ & 4.110 & $3.818-41.258$ & 0.005 \\
3 & $11(3.8)$ & 12.550 & 0.001 \\
\hline
\end{tabular}

HR, hazard ratio; CI, confidence interval.

*The risk factors are defined as an age at surgery of less than 16, the presence of perianal disease at surgery, and colonic or ileocolonic location at surgery. 
Table 6. Surgical Recurrence Outcome According to Risk Factors

\begin{tabular}{|c|c|c|c|}
\hline Variable & $\begin{array}{l}\text { Low risk group } \\
\text { (0 or } 1 \text { risk factor*) }\end{array}$ & $\begin{array}{l}\text { High risk group } \\
\text { (2 or } 3 \text { risk factors) }\end{array}$ & p-value \\
\hline No. & 203 (69.5) & 89 (30.5) & \\
\hline Follow-up duration, mo & $44.0(23.0-71.0)$ & $50.0(26.0-77.5)$ & 0.267 \\
\hline Surgical recurrence & $25(12.3)$ & $25(28.1)$ & 0.001 \\
\hline Time to surgical recurrence, mo & $103.4(96.7-111.3)$ & $84.3(73.3-95.3)$ & 0.001 \\
\hline
\end{tabular}

Data are presented as number (\%) or median (interquartile range).

*The risk factors are defined as an age at surgery of less than 16, the presence of perianal disease at surgery, and colonic or ileocolonic location at surgery.

\section{DISCUSSION}

The rapidly increasing tendency with complicated disease behavior over the past few decades and postoperative recurrence are crucial issues of CD management in Korea. ${ }^{1,11}$ Eastern Asian patients, including Koreans, have shown different clinical characteristics and disease courses from Caucasian CD patients. ${ }^{9}$ In this study, we identified the clinical characteristics and longterm outcomes in a large number of Korean patients from multiple referral centers.

The differences between our study patients from Western CD patients were male predominance (67.8\%), a small number of patients who were diagnosed CD after age 40 (11.3\%), smaller proportion of smokers and less frequent colonic involvement at surgery, and more common presence of perianal disease before surgery. ${ }^{9,14}$ The reason for these differences is mostly unknown. However, it is suggested that altered gut microbiota and genetic differences, such as no association with NOD2 and more strong association with TNFSF 15 compared with Caucasian, may be related. ${ }^{15}$ Higher exposure to social life or Westernized life style in Asian males compared to females might contribute to male predominance. Also, age-specific environmental factors or different sensitivities to certain infectious factors might cause a second small peak onset of $\mathrm{CD} .{ }^{16,17}$

Previously, the surgical recurrence rate in Western CD patients was 20\%-50\% compared to $14 \%-25 \%$ in Korean CD patients including present study within 5 years. ${ }^{5,18}$ Lower recurrence rate in Korean patients is might be due to the lower smoking rate and the genetic difference making less aggressive natural course compared to Western $\mathrm{CD}$ patients. ${ }^{13}$ However, the surgical recurrence rate in Western CD patients showed a wide range depending on the time period. Moreover, some recent studies conducted in the West showed only $8.6 \%$ within 8 years and $19.1 \%$ in 10 years. ${ }^{19,20}$ Therefore, it is still unclear to consider the ethnic difference as a risk factor for surgical recurrence.

Regarding medical treatment, 55.1\% of patients used thiopurine and only $19.9 \%$ of patients used anti-TNF medication before their first intestinal resection surgery. These results were similar to a recent single-center retrospective study. ${ }^{11}$ A tendency towards the lower proportion of anti-TNF agents in Asian countries compared to the West was well described in several studies. Although the efficacy of anti-TNF agents did not differ between the West and Asian countries, the lower proportion of usage may be originated from limited and strict indications under national insurance coverage in our study period in Korea. In this study, independent risk factors for postoperative surgical recurrence were younger age, colonic involvement, and the presence of perianal lesion at the surgery. Especially, patients who had more than 2 risk factors showed higher surgical recurrence. Thiopurine medication after surgery reduced surgical recurrence significantly. Patients with age under 16 at surgery showed much higher surgical recurrence than those with age over 16, especially compared to those with age over 40 . Recent American Gastroenterological Association review also suggested younger age at onset as one of the risk factors for postoperative recurrence. ${ }^{7}$ It was indicated that patients with younger age at onset have more extensive disease involvement and aggressive clinical course characteristics distinct from adult-onset patients. ${ }^{21-23}$ Indeed, our study population revealed more frequent colonic involvement and penetrating behavior compared to adult-onset patients. A different pathogenic mechanism such as the presence of a specific mutation in genes encoding the IL10R between the age groups may be related. ${ }^{24}$

There were conflicting results regarding disease location and surgical recurrence. In our study, a total of 34 patients (25.6\%) with colonic involvement showed surgical recurrence with a shorter median duration of 111.0 months compared to 119.0 months in patients with ileal location. It may be originated from involved bowel length, but our study was limited due to lack of surgical data. Some Western studies suggested that ileal location of $\mathrm{CD}$ is a predictive factor. ${ }^{25}$ But other studies showed colonic or ileocolonic location is related to higher recurrence rates compared the ileal disease. ${ }^{26,27}$ Also, in previous studies conducted in Korea, the relationship between location and surgical recurrence has not been clear. ${ }^{11}$

Patients with perianal disease are related to complicated disease phenotype, higher risk of surgery, hospitalization, and severe disease courses. ${ }^{28}$ Also, presence of perianal disease is a well-known risk factor for postoperative recurrence. ${ }^{6}$ A recent study conducted in Korea demonstrated that patients with peri- 
anal disease had increased risks of abdominal reoperation (HR, 1.978; 95\% CI, 1.034 to 3.784), and postoperative 5-ASA (HR, 0.388 ; $95 \%$ CI, 0.175 to 0.858 ) and thiopurine medication (HR, 0.499 ; $95 \%$ CI, 0.249 to 0.999 ) reduced risks of abdominal reoperation. ${ }^{29}$ The present study showed similar results in regard of perianal disease and benefit of postoperative thiopurine use. Also, patients with younger age at onset showed a higher rate of perianal disease in both studies. However, unlike the previous study, this study showed younger age was one of the risk factors and postoperative 5-ASA medication was not effective to prevent a recurrence.

Many studies from Western countries have shown that smoking is a strong risk factor for the postoperative recurrence of CD. ${ }^{4,6,7}$ Smoking rate in general population in Korea (19.9\% to 25.9\%) is comparable or higher than that of Western countries $(18.4 \%$ to $20.7 \%){ }^{30}$ However, a relatively small number of patients were current smokers at the time of diagnosis $(n=51$, $17.5 \%)$ and surgery $(n=40,13.7 \%)$ in the present study. This result was consistent with population-based cohort studies in Asian patients with CD that showed lower smoking rate (11.8\% to $28.0 \%$ ) than Western (57.0\%) and Eastern European patients $(62.0 \%)^{31}$

Another important finding was that thiopurine medication after surgery significantly reduced surgical recurrence. However, other medications including anti-TNF agents were not associated with surgical recurrence in our study. The top-down strategy of using an anti-TNF agent early in the postoperative period, as well as early in the disease course, has been proposed. ${ }^{2}$ Recently, several studies conducted in Asia showed contrary results from the present study. ${ }^{11,32}$ During our follow-up periods, the use of anti-TNF agents, both infliximab and adalimumab, were covered by national insurance whenever fulfilling following criteria of unresponsive effect with more than 2 drugs or moderate CD with contraindication to corticosteroid or immunomodulator. Therefore, a considerable number of patients could use anti-TNF medications after marked endoscopic and clinical recurrence. As a result, using anti-TNF medication still could not prevent a surgical recurrence.

There are several limitations in the present study. First, we could not evaluate endoscopic recurrence and clinical recurrence. Because of the study design which was multicenter and retrospective, there was no universal follow-up protocol. Therefore, we acquired only small number of endoscopic followup results and clinical data such as Crohn's Disease Activity Index, admission or medication step-up due to CD exacerbation. Second, we had limited data collection for histological and surgical-related factors that may be associated with surgical recurrence. Nevertheless, this study has the significance that it is the first study involving most of the referral centers nationwide, thus reflecting the overall results of Korean patients.

In conclusion, patients with younger age at onset, colonic location, and the presence of perianal disease need careful monitoring and intensive management strategies. Thiopurine medication in postoperative patients might be effective methods for the prevention of surgical recurrence.

\section{CONFLICTS OF INTEREST}

No potential conflict of interest relevant to this article was reported.

\section{ACKNOWLEDGEMENTS}

This work supported by Wonkwang University 2018.

\section{AUTHOR CONTRIBUTIONS}

Conceptualization: J.H.C., G.S.S., B.I.J. Data curation: S.B.K., J.H.C., G.S.S., B.I.J. Formal analysis: J.M.L., S.B.K., G.S.S. Investigation: S.B.K., J.H.C., G.S.S., B.I.J. Methodology and project administration: J.H.C., G.S.S., B.I.J. Resources: J.H.C., J.J.P., E.S.K., S.W.J., S.A.J., D.I.P., C.K.L., J.P.I., Y.S.K., H.S.K., J.L., C.S.E., B.I.J., G.S.S. Writing - original draft: S.B.K. Writing - review \& editing: G.S.S., B.I.J.

\section{ORCID}

$\begin{array}{ll}\text { Sung Bae Kim } & \text { https://orcid.org/0000-0002-7692-466X } \\ \text { Jae Hee Cheon } & \text { https://orcid.org/0000-0002-2282-8904 } \\ \text { Jae Jun Park } & \text { https://orcid.org/0000-0001-9974-1658 } \\ \text { Eun Soo Kim } & \text { https://orcid.org/0000-0003-0806-9136 } \\ \text { Seong Woo Jeon } & \text { https://orcid.org/0000-0002-9539-9389 } \\ \text { Sung-Ae Jung } & \text { https://orcid.org/0000-0001-7224-2867 } \\ \text { Dong Il Park } & \text { https://orcid.org/0000-0003-2307-8575 } \\ \text { Chang Kyun Lee } & \text { https://orcid.org/0000-0002-4279-3825 } \\ \text { Jong Pil Im } & \text { https://orcid.org/0000-0003-1584-0160 } \\ \text { You Sun Kim } & \text { https://orcid.org/0000-0002-5156-3458 } \\ \text { Hyun Soo Kim } & \text { https://orcid.org/0000-0003-4834-0496 } \\ \text { Jun Lee } & \text { https://orcid.org/0000-0002-8060-9646 } \\ \text { Chang Soo Eun } & \text { https://orcid.org/0000-0001-6533-9644 } \\ \text { Jeong Mi Lee } & \text { https://orcid.org/0000-0003-0878-5802 } \\ \text { Byung Ik Jang } & \text { https://orcid.org/0000-0002-3037-9272 } \\ \text { Geom Seog Seo } & \text { https://orcid.org/0000-0001-8789-7989 }\end{array}$

\section{REFERENCES}

1. Park JJ, Yang SK, Ye BD, et al. Second Korean guidelines for the management of Crohn's disease. Korean J Gastroenterol 2017;69:29-54

2. Lichtenstein GR, Loftus EV, Isaacs KL, Regueiro MD, Gerson LB, Sands BE. ACG clinical guideline: management of Crohn's disease in adults. Am J Gastroenterol 2018;113:481-517.

3. Rungoe C, Langholz E, Andersson M, et al. Changes in medical treatment and surgery rates in inflammatory bowel disease: a na- 
tionwide cohort study 1979-2011. Gut 2014;63:1607-1616.

4. Gklavas A, Dellaportas D, Papaconstantinou I. Risk factors for postoperative recurrence of Crohn's disease with emphasis on surgical predictors. Ann Gastroenterol 2017;30:598-612.

5. Blum E, Katz JA. Postoperative therapy for Crohn's disease. Inflamm Bowel Dis 2009;15:463-472.

6. Cohen-Mekelburg S, Schneider Y, Gold S, Scherl E, Steinlauf A. Risk stratification for prevention of recurrence of postoperative Crohn's disease. Gastroenterol Hepatol (N Y) 2017;13:651-658.

7. Regueiro M, Velayos F, Greer JB, et al. American Gastroenterological Association Institute technical review on the management of Crohn's disease after surgical resection. Gastroenterology 2017;152:277-295.

8. De Cruz P, Kamm MA, Hamilton AL, et al. Crohn's disease management after intestinal resection: a randomised trial. Lancet 2015;385:1406-1417.

9. Park SJ, Kim WH, Cheon JH. Clinical characteristics and treatment of inflammatory bowel disease: a comparison of Eastern and Western perspectives. World J Gastroenterol 2014;20:1152511537.

10. Ng SC. Emerging leadership lecture: inflammatory bowel disease in Asia: emergence of a "Western" disease. J Gastroenterol Hepatol 2015;30:440-445.

11. Yang KM, Yu CS, Lee JL, et al. Risk factors for postoperative recurrence after primary bowel resection in patients with Crohn's disease. World J Gastroenterol 2017;23:7016-7024.

12. Lee KY, Yu CS, Lee KY, et al. Risk factors for repeat abdominal surgery in Korean patients with Crohn's disease: a multi-center study of a Korean inflammatory bowel disease study group. J Korean Soc Coloproctol 2012;28:188-194.

13. Lee YW, Lee KM, Chung WC, Paik CN, Sung HJ, Oh YS. Clinical and endoscopic recurrence after surgical resection in patients with Crohn's disease. Intest Res 2014;12:117-123.

14. Ng SC, Tang W, Ching JY, et al. Incidence and phenotype of inflammatory bowel disease based on results from the Asiapacific Crohn's and colitis epidemiology study. Gastroenterology 2013;145:158-165.

15. Cheon JH. Genetics of inflammatory bowel diseases: a comparison between Western and Eastern perspectives. J Gastroenterol Hepatol 2013;28:220-226

16. Ananthakrishnan AN. Epidemiology and risk factors for IBD. Nat Rev Gastroenterol Hepatol 2015;12:205-217.

17. Li X, Song P, Li J, et al. The disease burden and clinical characteristics of inflammatory bowel disease in the Chinese population: a systematic review and meta-analysis. Int J Environ Res Public Health 2017;14:E238.

18. Kristo I, Stift A, Bergmann M, Riss S. Surgical recurrence in Crohn's disease: are we getting better? World J Gastroenterol 2015;21:6097-6100
19. Riss S, Schuster I, Papay P, et al. Surgical recurrence after primary ileocolic resection for Crohn's disease. Tech Coloproctol 2014;18:365-371.

20. de Buck van Overstraeten A, Eshuis EJ, Vermeire S, et al. Shortand medium-term outcomes following primary ileocaecal resection for Crohn's disease in two specialist centres. Br J Surg 2017;104:1713-1722.

21. Peyrin-Biroulet L, Loftus EV Jr, Colombel JF, Sandborn WJ. The natural history of adult Crohn's disease in population-based cohorts. Am J Gastroenterol 2010;105:289-297.

22. Duricova D, Fumery M, Annese V, Lakatos PL, Peyrin-Biroulet L, Gower-Rousseau C. The natural history of Crohn's disease in children: a review of population-based studies. Eur J Gastroenterol Hepatol 2017;29:125-134.

23. Thia KT, Luman W, Jin OC. Crohn's disease runs a more aggressive course in young Asian patients. Inflamm Bowel Dis 2006;12:5761.

24. Abraham BP, Mehta S, El-Serag HB. Natural history of pediatriconset inflammatory bowel disease: a systematic review. J Clin Gastroenterol 2012;46:581-589.

25. Manser CN, Frei P, Grandinetti T, et al. Risk factors for repetitive ileocolic resection in patients with Crohn's disease: results of an observational cohort study. Inflamm Bowel Dis 2014;20:15481554

26. Morar PS, Faiz 0, Hodgkinson JD, et al. Concomitant colonic disease (Montreal L3) and re-resectional surgery are predictors of clinical recurrence following ileocolonic resection for Crohn's disease. Colorectal Dis 2015;17:0247-0255

27. Kim HJ, Oh SH, Kim DY, et al. Clinical characteristics and longterm outcomes of paediatric Crohn's disease: a single-centre experience. J Crohns Colitis 2017;11:157-164.

28. Zhao M, Lo BZS, Vester-Andersen MK, Vind I, Bendtsen F, Burisch J. A 10-year follow-up study of the natural history of perianal Crohn's disease in a Danish population-based inception cohort. Inflamm Bowel Dis 2019;25:1227-1236.

29. Han YM, Kim JW, Koh SJ, et al. Patients with perianal Crohn's disease have poor disease outcomes after primary bowel resection. J Gastroenterol Hepatol 2016;31:1436-1442.

30. Prideaux L, Kamm MA, De Cruz P, et al. Comparison of clinical characteristics and management of inflammatory bowel disease in Hong Kong versus Melbourne. J Gastroenterol Hepatol 2012;27:919-927.

31. Ng SC, Bernstein CN, Vatn MH, et al. Geographical variability and environmental risk factors in inflammatory bowel disease. Gut 2013;62:630-649.

32. Kusaka J, Shiga H, Kuroha M, et al. Risk factors associated with postoperative recurrence and repeat surgery in Japanese patients with Crohn's disease. Int J Colorectal Dis 2017;32:1407-1413. 